\title{
QUIROSOLITONES RCP Y LCP EN ÓPTICA Y MICROONDAS EN RÉGIMEN NORMAL Y DE METAMATERIAL
}

\author{
Mario Zamorano L. ${ }^{1} \quad$ Héctor Torres $\mathbf{S} .^{1}$ \\ Recibido el 16 de julio de 2003, aceptado el 8 de marzo de 2004
}

\begin{abstract}
RESUMEN
En este artículo se presenta la solución de las ecuaciones de Maxwell para pulsos electromagnéticos de polarización circular a la derecha (RCP) y de polarización circular a la izquierda (LCP) en régimen normal y de metamaterial. El medio de propagación corresponde a un medio dispersivo, quiral y no lineal. El efecto quiral se caracteriza a través del formalismo de Born-Fedorov y la no-linealidad considerada es del tipo Kerr. La simulación de los modelos presentados se realiza mediante el método split-step de Fourier y los resultados obtenidos muestran que para ambos tipos de pulsos, RCP y LCP, hay un efecto compensador entre la atenuación del medio y el efecto quiral.
\end{abstract}

Palabras claves: Ecuaciones de Maxwell, quiralidad, polarización circular, metamaterial.

\begin{abstract}
This paper presents the solution of Maxwell's equations for electromagnetic pulses with right circular polarization $(R C P)$ and left circular polarization $(L C P)$ in normal and metamaterial regimes. The propagating medium is dispersive, chiral and nonlinear. The chirality effect is characterized through Born-Fedorov formalism. The nonlinearity used is of a Kerr type. Models simulation is carried out through split-step Fourier method and the results show that for both types of pulses, there is a compensating, effect between the medium attenuation and the chiral factor.
\end{abstract}

Keywords: Maxwell equations, chirality, circularly polarization, metamaterial.

\section{INTRODUCCIÓN}

La actividad óptica natural es tal vez una de las propiedades más conocida pero menos comprendida. Aunque es tratada en muchos textos rara vez se explica más allá de que su observación está relacionada con la birrefringencia circular y la falta de simetría tipo espejo. El interés por los medios quirales es de antigua data en química y biología y recientemente han surgido otros campos de investigación, como la propagación de ondas electromagnéticas tanto en la región óptica como de microondas. La actividad óptica sólo se observa en medios que contienen moléculas quirales o, más generalmente, en estructuras asimétricas cuya simetría de grupo no contiene el elemento $S_{n}$ - una rotación en un ángulo $2 \pi / n$ alrededor de un eje dado seguida por una reflexión en un plano perpendicular a dicho eje. En particular, no contiene el elemento $S_{1}$ (un plano de simetría espejo) y $S_{2}$ (un punto de simetría de inversión).

La quiralidad en moléculas lleva a un gran número de interesantes estructuras, algunas muy simples y regulares (cristales) y otras de gran complejidad (sistemas biológicos). Existen, en el campo óptico, modelos simples para estudiar estructuras quirales, en los cuales los medios periódicos pueden ser tratados adecuadamente como homogéneos en términos macroscópicos, como es usual por ejemplo para cristales sólidos. La descripción de las propiedades macroscópicas de cristales es un problema clásico en óptica [1]. Desde este punto de vista el interés en el uso de pequeñas espiras (fases helicoidales LC), para modificar las propiedades del medio, está relacionado con el hecho de que es posible usar, como punto de partida para nuestro análisis, el universalmente conocido modelo usado donde la estructura molecular es despreciada y el medio es tratado como un continuo.

\footnotetext{
1 Universidad de Tarapacá, Departamento de Electrónica, Casilla 6-D, Arica-Chile, zamorano@uta.cl, htorres@uta.cl
} 
Este modelo, que es no-homogéneo y periódico, se denominará mesoscópico y el modelo homogéneo, correspondiente a un cristal perfecto, se denominará macroscópico. El objetivo principal de nuestro trabajo es la definición de modelos macroscópicos para el estudio, simulación y análisis de la propagación de ondas RCP y LCP en medios quirales en el rango óptico y de microondas.

Para este propósito hay que observar la existencia de muchos problemas, en la óptica de cristales quirales, que aún no han sido resueltos, ya que las propiedades ópticas de tales medios están fuertemente incorporadas en el marco de modelos homogéneos. Los principales problemas se derivan de la asimetría intrínseca de estos medios y de la naturaleza no local de la actividad óptica, que están reflejadas en la gran cantidad de ecuaciones constitutivas usadas para describir medios ópticamente activos. Cualquier conjunto de ecuaciones constitutivas define un posible modelo macroscópico para estructuras periódicas reales. La relación entre los diferentes modelos ha sido discutida largamente en la literatura especializada [2], pero solamente en el último decenio ha sido más o menos bien esclarecida [3], [4], [5].

En este artículo se describe un modelo de propagación de solitones en un medio quiral. El modelo considera la dispersión temporal, la dispersión espacial (quiralidad), la atenuación y las no-linealidades del medio. Los resultados presentados aquí se enmarcan en la línea de investigaciones sobre materiales electromagnéticos complejos. En otros artículos [6] se ha presentado una teoría fenomenológica que describe la auto-acción de los pulsos electromagnéticos en ciertos materiales quirales. La teoría está basada en el formalismo de Beltrami-Maxwell que ha sido extendido para medios quirales no lineales [7], [8].

En la sección siguiente se resume la base teórica del trabajo, una extensión de artículos recientes sobre solitones quirales. Luego se presenta la discusión de los resultados numéricos y finalmente se plantean algunas conclusiones.

\section{ASPECTOS TEÓRICOS}

En esta sección se resumen los aspectos teóricos esenciales de la propagación de solitones quirales, discutidos ampliamente en las referencias [8] y [9].

\section{Actividad Óptica Natural y Hélices}

Dentro de un medio isotrópico quiral, los vectores de campo de la luz describen una hélice. Como fue demostrado por Fresnel, esto es consecuencia del hecho que los índices de refracción de ondas polarizadas circularmente a la derecha (RCP) y a la izquierda (LCP) son ligeramente diferentes (dicroismo circular). Estos mismos vectores describen también si consideramos luz polarizada circularmente. Considerando estos hechos, Fresnel propone, por analogía, que la forma estereoquímica de cristales quirales y, en particular los cristales de cuarzo, es esencialmente helicoidal [10].

El modelo helicoidal para la actividad óptica fue sugerido primero por Fresnel y ha llegado a ser cada vez más importante. Drude [11] explica la actividad óptica asumiendo que los electrones, dentro de las moléculas quirales ,oscilan a lo largo como una hélice. Esto determina que el vector de polarización $P$ contenga un término proporcional al rotor de $E$. En unidades MKS, las ecuaciones constitutivas son

$$
\begin{gathered}
\vec{D}=\varepsilon(\vec{E}+T \nabla \times \vec{E}) \\
B=\mu H
\end{gathered}
$$

En las ecuaciones anteriores tanto $\varepsilon$ como $\mu$ son escalares y el factor quiral $T$ tiene unidades de longitud. Haciendo una aproximación gruesa podemos estimar que, en el rango de las frecuencias ópticas, el factor quiral tendrá las dimensiones del grupo molecular quiral.. La birrefringencia circular, definida como la diferencia, $\Delta n$, entre los índices de refracción de ondas polarizadas circularmente a la derecha e izquierda, es generalmente pequeña y está dada por

$$
\Delta n=2 \pi T / \lambda
$$

donde $\lambda$ es la longitud de onda en el interior del medio. La "potencia rotatoria", es decir la rotación del plano de polarización por unidad de trayectoria del haz linealmente polarizado, es

$$
\rho=\frac{2 \pi^{2}}{n} \frac{T}{\lambda^{2}}
$$

donde $n=\sqrt{\varepsilon}$.

Los parámetros quirales $T$ y $\rho$ son los parámetros ópticos más importantes, explícitamente dependientes de la magnitud molecular. Las leyes de escalamiento, dadas por las ecuaciones (2) y (3), fueron planteadas primeramente por Boltzmann [12], sobre la base de un modelo molecular simple. Sin embargo, se observa, de acuerdo con el modelo de electrones oscilantes de Drude, que los parámetros $\varepsilon$ y $T$ dependen de la , Chile, Vol. $12 N^{o} 1,2004$ 
longitud de onda de la luz debido a la dispersión de frecuencia. Esto implica una dependencia más complicada de $\Delta n$ y $\rho$ de $\lambda$. Para un modelo de Drude de electrón único, el parámetro $T$ cambia su signo a la frecuencia propia del electrón oscilante dando una dependencia de $\rho$ sobre $\lambda$ del tipo $\left(\lambda^{2}-\lambda_{1}^{2}\right)^{-1}$, donde $\lambda_{1}$ es la longitud de onda correspondiente a la frecuencia propia. En tratamientos de mecánica cuántica el número de frecuencias propias llega a ser infinito.

Una interesante realización del modelo de Drude fue hecha por Lindman [13]. Él demostró que una pequeña caja que contenía pequeñas hélices de alambre de cobre, distribuidas aleatoriamente, producía la rotación del plano de polarización de un haz de microondas.. La oscilación de los electrones de conducción dentro del material puede ser tratada clásicamente y la dispersión óptica por rotación cumple con la ecuación de Drude.

Desde hace unos años, la computación digital ha abierto una nueva área de investigación sobre la dispersión por hélices [3], [4], y sus resultados han sido de gran ayuda para comprender mejor la actividad óptica natural en términos del "modelo de hélice". La mayoría de las moléculas ópticamente activas tienen una conformación helicoidal. En otras moléculas quirales la disposición de sus átomos o grupos de átomos, puede ser usada para definir un conjunto helicoidal hacia la derecha ó izquierda, lo que gobierna la actividad óptica [14], [15].

\section{Ecuaciones Constitutivas}

La actividad óptica natural está relacionada con las derivadas espaciales del campo eléctrico que aparece en la ecuación (1), lo que da origen a la dispersión espacial. En otras palabras, la actividad óptica es una propiedad no local que deriva de la estructura molecular de la materia o, más generalmente, de la no uniformidad espacial [16].

Las ecuaciones constitutivas (1) son fácilmente generalizadas a medios anisotrópicos, considerando las cantidades $\varepsilon$ y $T$ como tensores de segundo rango. Una generalización ulterior se logra considerando derivadas espaciales de más alto orden. Los inconvenientes presentados por las Ecs. (1) son: a) los materiales caracterizados por ellas son no recíprocos y b) no cumplen con las condiciones de borde. Las ecuaciones de Born-Fedorov superan los problemas anteriores [17], y están dadas por

$$
\vec{D}=\varepsilon(\vec{E}+T \nabla \times \vec{E})
$$

$$
\vec{B}=\mu_{0}(\vec{H}+T \nabla \times \vec{H})
$$

En nuestra contribución se considera además que el medio es no-lineal, por lo que la Ec. (4a) se transforma en

$$
\vec{D}=\varepsilon_{n} \vec{E}+\varepsilon T \nabla \times \vec{E}
$$

La no-linealidad es de tipo Kerr, definida por un índice de refracción tal que la permitividad es [18]

$$
\varepsilon_{n}=\varepsilon+\varepsilon_{2}|\vec{E}|^{2}
$$

donde $\varepsilon$ es la parte lineal y $\varepsilon_{2}$ es la parte no lineal, respectivamente, de $\varepsilon_{n}$. Se ha demostrado en trabajos anteriores, [8], [9], que, a partir de las ecuaciones de Maxwell y para las ecuaciones constitutivas anteriores, la ecuación de onda resultante es la siguiente

$$
\begin{aligned}
& \nabla^{2} \vec{E}+\mu \varepsilon T^{2} \frac{\partial \vec{E}^{2}}{\partial t^{\prime 2}} \nabla^{2} \vec{E}-\mu \varepsilon \frac{\partial \vec{E}^{2}}{\partial t^{\prime 2}}- \\
& 2 \mu \varepsilon T \nabla \times \frac{\partial \vec{E}^{2}}{\partial t^{\prime 2}}=\mu \varepsilon_{2}|\vec{E}|^{2} \frac{\partial \vec{E}^{2}}{\partial t^{\prime 2}}+ \\
& \mu \varepsilon_{2} T|\vec{E}|^{2} \nabla \times \frac{\partial \vec{E}^{2}}{\partial t^{\prime 2}}+\mu \sigma T \nabla \times \frac{\partial \vec{E}}{\partial t^{\prime}}+\mu \sigma \frac{\partial \vec{E}}{\partial t^{\prime}}
\end{aligned}
$$

donde $\sigma$ es la conductividad del medio y $t^{\prime}=t+(1 \pm k T) z / v_{g}$, siendo $v_{g}$ la velocidad de grupo. La solución homogénea de la ecuación (7) $\left(\varepsilon_{2}=0, \sigma=0\right)$ permite obtener la relación de dispersión $k^{2}=\omega_{0}^{2} \mu(1-k T) \varepsilon(1-k T)=\omega_{0}^{2} \mu_{e f} \varepsilon_{e f}$, de la cual, para $k T>1$, resulta una permitividad y una permeabilidad efectivas negativas $\left(\varepsilon_{e f}\right.$ y $\mu_{e f}$ respectivamente), y, por lo tanto, el medio se define como metamaterial o material LHM (Left Handed Medium) [19].

Suponiendo que el campo eléctrico óptico $\vec{E}$ representa una onda polarizada hacia la derecha $(+)$ o hacia la izquierda (-) y que se propaga en la dirección z, se tendrá que 


$$
\begin{aligned}
& \left.\vec{E}_{ \pm}\left(\vec{r}, t^{\prime}\right)=\hat{x} \pm \hat{y} \Psi_{ \pm} \vec{x} t^{\prime} e\right)^{j\left(k_{ \pm} z^{*}-\omega_{0} t^{\prime}\right)} \\
& =\vec{\Psi}_{ \pm} e^{-j\left(k_{ \pm} z^{*}-\omega_{0} t^{\prime}\right)}
\end{aligned}
$$

donde $\omega_{0}$ es la frecuencia de la portadora óptica y $z^{*}=z /\left(1-k^{2} T^{2}\right)$. Remplazando la ecuación (8) en la ecuación (7) da como resultado

$$
\begin{aligned}
& \frac{\partial \phi}{\partial z}-j(1 \pm T k) \frac{1}{2} k^{\prime} \frac{\partial^{2} \phi}{\partial t^{2}}-\left(\# T k \frac{3}{6} k \frac{\partial^{3} \phi}{\partial t^{3}}\right. \\
& +\frac{\omega_{0} \alpha}{2 k_{0}} \phi+j \frac{\beta \omega_{0}^{2}}{2 k_{0}}|\phi|^{2} \phi=0
\end{aligned}
$$

en (8) $\vec{\Psi}_{ \pm}$representa la envolvente compleja para una onda circularmente polarizada a la derecha RCP (+) ó a la izquierda LCP (-). Además

$$
\begin{aligned}
& t=t^{\prime}-\left(\mathbb{E} T k \quad N_{g} ; k \doteq \frac{\partial k}{\partial \omega}=\frac{1}{v_{g}}\right. \\
& k^{\prime}=\frac{\partial^{2} k}{\partial \omega^{2}} \quad ; k=\frac{\partial^{3} k}{\partial \omega^{3}} \quad \vec{\Psi}_{{ }_{ \pm}}=\phi \\
& \beta=\mu \varepsilon_{2} ; z=z^{*} /\left(1-T^{2} k_{0}^{2}\right) \\
& k_{+}=\frac{1}{1+T k_{0}} \quad ; \quad k_{-}=\frac{1}{1-T k_{0}} \\
& v^{2}=\frac{1}{\mu \varepsilon} \quad ; \quad \alpha=\mu \sigma \quad ; \quad k_{0}=\frac{\omega_{0}}{c}
\end{aligned}
$$

Si se trabaja en la tercera ventana óptica, $k^{\prime} \stackrel{\prime}{\prime}^{\prime}$, la ecuación (9) se transforma en

$$
\begin{aligned}
& \frac{\partial \phi}{\partial z}-j(1 \pm T k) \frac{1}{2} k^{\prime} \frac{\partial^{2} \phi}{\partial t^{2}}+\frac{\omega_{0} \alpha}{2 k_{0}} \phi \\
& +j \frac{\beta \omega_{0}^{2}}{2 k_{0}}|\phi|^{2} \phi=0
\end{aligned}
$$

La ecuación (10) modela la propagación de pulsos en medios quirales, dispersivos, con pérdidas y no lineales.

Esta ecuación describe, por ejemplo, la propagación de pulsos RCP o LCP en medios quirales, tanto en la región óptica como en la de microondas. En la región óptica el análisis de cada término es el siguiente: El término de la izquierda representa la evolución espacial del pulso con la distancia; el primer término de la derecha representa las pérdidas; el segundo término es la dispersión de la velocidad de grupo (GVD) y corresponde a la dispersión cromática del medio; y el tercero es el término no-lineal y se debe al efecto Kerr, [8], [9]. En el modelo presentado la quiralidad afecta al término de la dispersión cromática mediante el factor $(1 \pm T k)$. El grado de importancia de los términos de la ecuación (10) dependerá, entre otros, del ancho inicial y de la potencia "peca" del pulso de entrada y determinará la evolución del pulso dentro del medio.

En la versión de microondas, la ecuación (10) también constituye un buen modelo para un bioplasma con pérdidas que simule el comportamiento electromagnético de la cabeza humana irradiada por teléfonos celulares, ya que las autofrecuencias de excitaciones colectivas tipo "twist" (o hélices) en cadenas moleculares [20] están en el rango de gigahertz. Las cadenas de DNA, que son hélices dobles a la derecha o izquierda inmersas en un ambiente acuoso, donde además se tiene un conjunto de proteínas, membranas, etc. que representan el bioplasma, poseen una quiralidad geométrica que puede ser representada por el factor $T$. En este caso, la velocidad de la onda es mucho menor debido a la alta constante dieléctrica del medio $(\sim 50 \varepsilon)$. Los términos más importantes, en la versión de microondas, son la atenuación $\left(\sigma \sim 1[\Omega / m]^{-1}\right)$, la dispersión cromática $(k ")$, la quiralidad y el término cúbico, el que, a frecuencias de microondas, como en el caso de un bioplasma, debe ser estudiado cuidadosamente. De acuerdo con la conjetura de Frolish [21], la energía en el material biológico puede ser transferida sin disipación si se forman dentro de las células estructuras solitónicas apropiadas. En nuestro modelo este efecto "sin disipación" se simula a través de la quiralidad si el factor $1-k T \rightarrow 0$ y por lo tanto $\alpha \rightarrow 0$. Por ello para el tratamiento de solitones en el rango de las microondas la ecuación (10) fue escalada en frecuencia, amplitud y duración del pulso de entrada.

Para efectos de este análisis, y para resolver posteriormente la ecuación (10), se sigue el procedimiento planteado en [18]. Por ello introducimos, primeramente, las escalas de longitud conocidas como longitud de dispersión $L_{D}$ y longitud no-lineal $L_{N L}$. A continuación se normalizan el tiempo y la amplitud de la señal de entrada

$$
\tau=\frac{t}{T_{0}}=\frac{t^{\prime}-\left(\Downarrow T k \Varangle k_{g}\right.}{T_{0}}
$$




$$
\phi(z, \tau)=\sqrt{P_{0}} \exp \left[-\frac{\alpha \omega_{0}(1 \pm T k) z}{2 k}\right] \vec{U}(z, \tau)
$$

donde $T_{0}$ y $P_{0}$ son, respectivamente, el ancho inicial y la potencia "peca" del pulso de entrada. Utilizando las ecuaciones (10)-(12) se demuestra que la amplitud normalizada $\vec{U}(z, \tau)$ satisface la ecuación de propagación dada por

$$
\begin{aligned}
& j \frac{\partial \vec{U}}{\partial z}=-\frac{\operatorname{sgn}\left(k^{\prime}\right.}{2 L_{D_{ \pm}}} \frac{\partial^{2} \vec{U}}{\partial \tau^{2}}+ \\
& \frac{\exp \left[-\alpha \omega_{0}(1 \pm T k) z / k\right]}{L_{N L}}|\vec{U}|^{2} \vec{U}
\end{aligned}
$$

donde $\operatorname{sgn}\left(k^{\prime} \ni \pm\right.$, dependiendo de si se trabaja en la región de dispersión normal o anómala, y

$$
\begin{aligned}
& L_{D_{ \pm}}=\frac{T_{0}^{2}}{\left|k^{\prime}\right|^{\prime} \notin T k} \\
& L_{N L}=\frac{2 k_{0}}{\omega_{0}^{2} \beta P_{0}}
\end{aligned}
$$

Dependiendo de las magnitudes relativas de $L_{D}, L_{N L}$ y de la longitud $L$ de la fibra óptica, la ecuación (10) definirá los siguientes regímenes de funcionamiento: a) régimen dispersivo, es decir que en la ecuación (10) domina el segundo término asociado a la dispersión de 1 velocidad de grupo (GVD); b) régimen no-lineal, en el cual domina el fenómeno de la automodulación de fase (SPM), definido por el tercer término de la ecuación (10); c) régimen dispersivo-no lineal, donde tanto la GVD y la SPM tienen importancia. En este trabajo hemos implementado este último régimen, a fin de estudiar el efecto de la quiralidad del medio sobre la propagación de pulsos tipo soliton. Para ello se normaliza la ecuación (10) [18]

$$
\begin{aligned}
& j \frac{\partial \vec{U}}{\partial \xi}=-\operatorname{sgn}\left(k^{\prime}, \frac{1}{2} \frac{\partial^{2} \vec{U}}{\partial \tau^{2}}+\right. \\
& N^{2} \exp \left[-\alpha \omega_{0}(1 \pm T k) z / k\right]|\vec{U}|^{2} \vec{U}
\end{aligned}
$$

donde $\xi$ y $\tau$ representan las variables distancia y tiempo, ambas normalizadas, dadas por

$$
\xi=\frac{z}{L_{D_{ \pm}}} ; \tau=\frac{t}{T_{0}}
$$

El parámetro $\mathrm{N}$ se define como la razón entre las longitudes de dispersión y no-lineal

$$
N^{2}=\frac{L_{D_{ \pm}}}{L_{N L}}=\frac{\omega_{0}^{2} \beta P_{0} T_{0}^{2}}{2 k_{0} \mid k^{\prime} \nmid(\boxplus T k}
$$

A través de la ecuación (10) o (16) se caracteriza la propagación de solitones en un medio dispersivo, nolineal, quiral y con pérdidas. Para este tipo de propagación la condición inicial está dada por [18]

$$
\phi(0, t)=\sqrt{P_{0}} \operatorname{senh}\left(t / T_{0}\right)
$$

Definiendo N, el orden del soliton, es posible determinar la potencia $P_{0}$ a partir de la ecuación (18)

$$
P_{0}=N^{2} \frac{2 k_{0} \mid k^{\prime} \nmid(\boxplus T k}{\omega_{0}^{2} \beta T_{0}^{2}}
$$

\section{ANÁLISIS DE RESULTADOS}

El modelado de la propagación de pulsos electromagnéticos RCP y LCP, en un medio dispersivo quiral y no lineal, dado en la sección anterior, se resuelve numéricamente a través del método espectral de Fourier (split-step) [22]. Las simulaciones se realizaron para régimen solitónico en un medio con quiralidad, la que se manifiesta a través del coeficiente quiral $(1-k T)$. Se presentan a continuación las condiciones que debe cumplir el coeficiente quiral para que el material sea normal ( $\varepsilon$ y $\mu$ positivos) o sea metamaterial ( $\varepsilon$ y $\mu$ negativos) [23], [24].

Régimen Normal: $k>0 ; k T<1$

$$
\begin{array}{ll}
R C P: & T>0 \\
L C P: & T<0
\end{array}
$$

Régimen Metamaterial: $k<0 ;|k T|>1$

$R C P: \quad T<0$

$L C P: T>0$ 
Las simulaciones realizadas se muestran en las Figs. 15. La primera corresponde al soliton inicial (referencial), es decir para una atenuación nula y $k T=0$. En la Fig. 2 se presenta el efecto que causa una atenuación de $5 d B$ sobre el soliton inicial. Las Figs. 3 y 4 muestran el efecto de un factor quiral negativo del medio de propagación sobre la amplitud de un quirosoliton RCP. Es esta respuesta la que permite compensar las pérdidas observadas en la Fig. 2. Lo mismo sucede con quirosolitones LCP, pero para un factor quiral positivo (Fig. 5). En las Figs. 6 y 7 se puede observar ejemplos del comportamiento de los quirosolitones RCP y LCP para una atenuación de $5 \mathrm{~dB}$ y factores quirales límites de 0 y \pm 2 respectivamente; el doble signo indica un mismo comportamiento para pulsos RCP con $k T=2$ y pulsos LCP con $k T=-2$. Para esos valores de quiralidad $k T= \pm 2$, el vector de onda está en dirección contraria al vector de Poynting del pulso, y corresponde al régimen de metamaterial

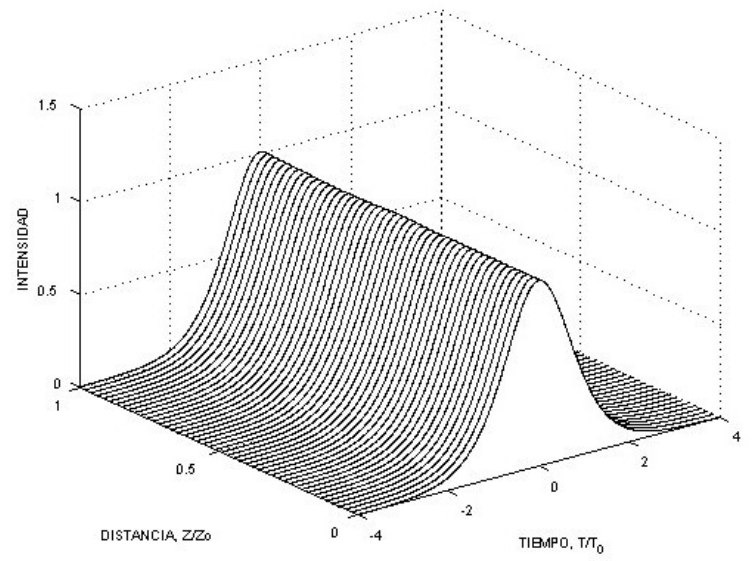

Fig. 1.- Soliton de referencia: Aten. $=0$ y kT=0

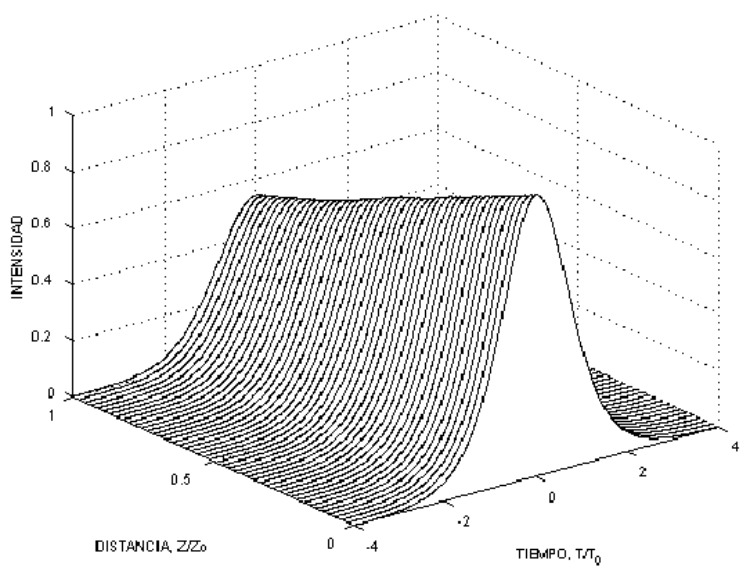

Fig. 2.- Soliton, $\mathrm{kT}=0 \mathrm{y}$ Aten. $=5 \mathrm{~dB}$

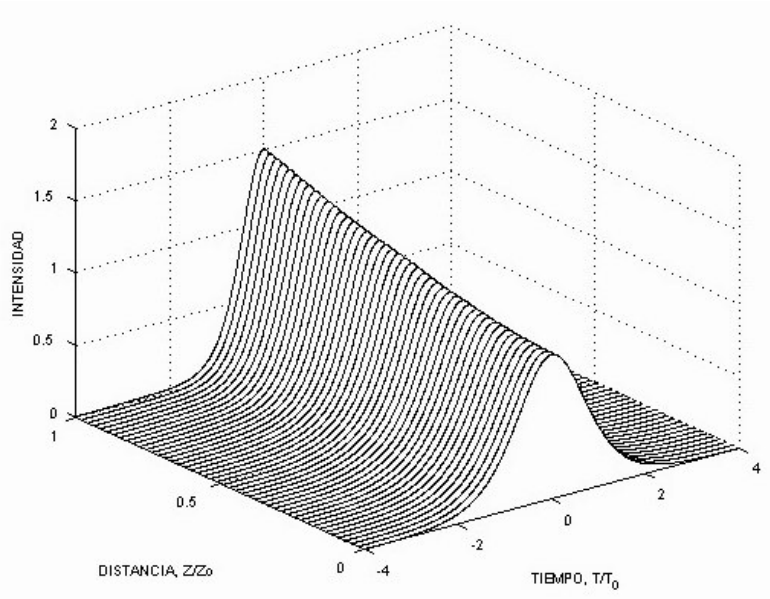

Fig. 3.- Quirosoliton RCP con Aten. $=0 \mathrm{~dB}$ y $\mathrm{kT}=-0.5$

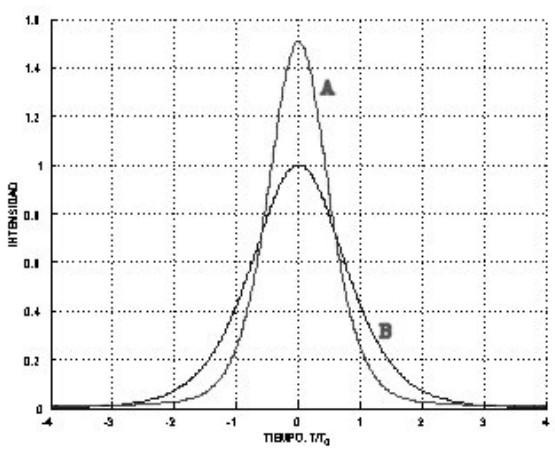

Fig. 4.- Perfiles inicial $\mathrm{B}$ y final $\mathrm{A}$ del quirosoliton RCP con Aten. $=1 \mathrm{~dB}$ y kT $=-0.5$

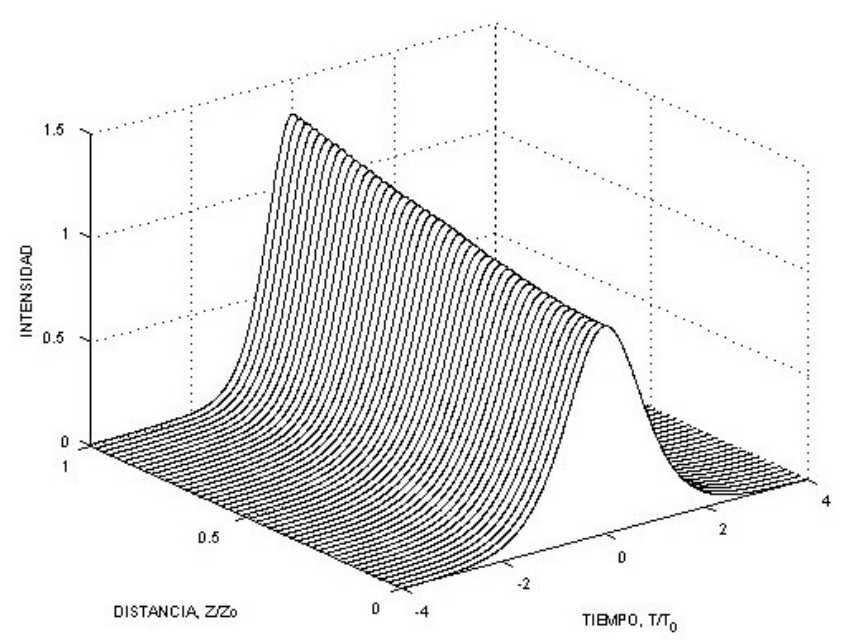

Fig. 5.- Quirosoliton LCP con Aten. $=0 \mathrm{~dB}$ y kT $=0.3$ 


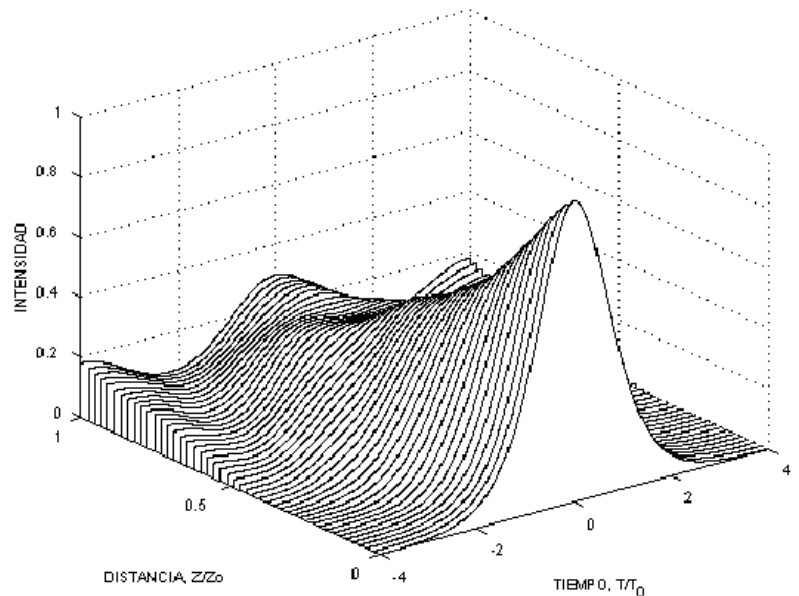

Fig. 6.- Quirosoliton RCP $k T=2$ Aten. $=5 \mathrm{~dB}$

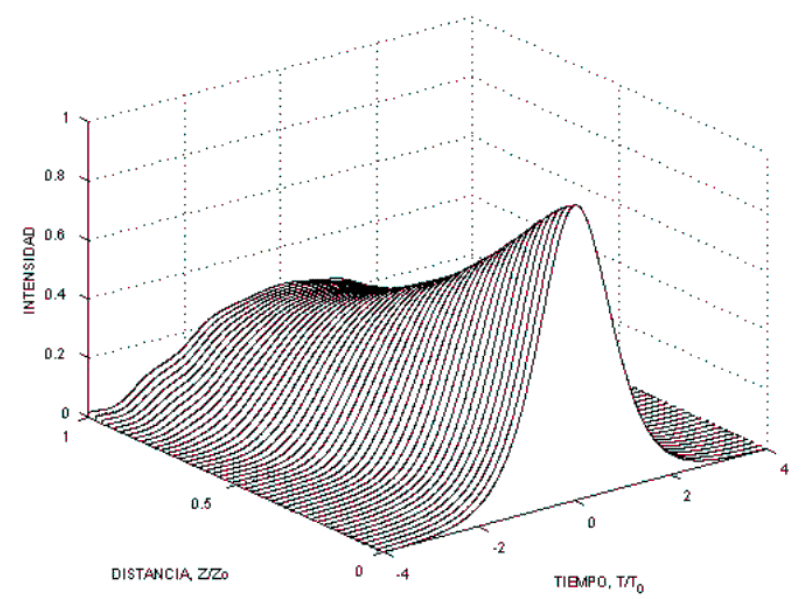

Fig. 7.- Quirosoliton LCP $k T=-2$ Aten. $=5 \mathrm{~dB}$

Mediante simulaciones sistemáticas, variando la atenuación y el factor quiral, es posible obtener curvas como las que se muestran en las Figs. 8 y 9, donde se observa la variación de la potencia de los pulsos en función de la quiralidad del medio de propagación, para diferentes valores de la atenuación. A fin de analizar adecuadamente la variación de los quirosolitones de primer orden RCP y LCP con el factor quiral, se ha representado, en las Figs. 8 y 9, la potencia óptica necesaria para la creación del soliton de primer orden, la que es de $0,87 \mathrm{~W}$.

El perfil que muestran las Figs. 8 y 9 está definido por el factor quiral, como se deduce de la Ec. (10). Así, por ejemplo, para un medio sin pérdidas $(\alpha=0)$ y cuando $k T=0$, ambos pulsos son iguales $\left(k_{+}=k_{-}\right)$, se trabaja en régimen solitónico convencional y la potencia del soliton será $P_{0}$. En este caso las longitudes de dispersión y no lineal son iguales, Ecs. (14) y respectivamente.

También se deduce de la Ec. (10) que para pulsos RCP una variación del factor $0>k T>-1$ significará un aumento en la potencia del soliton respecto de la referencia y pasará por un máximo. Lo mismo ocurre para pulsos LCP pero cuando el factor quiral varía $0<k T<1$. En este rango se tiene un régimen de funcionamiento dispersivo no-lineal.

Cuando para un pulso RCP se tiene $k T=-1$ y para un pulso LCP se tiene $k T=1$ se trabaja en régimen nolineal puro ya que el término dispersivo se anula (la longitud de dispersión, Ec. (14), tiende a infinito).

Finalmente, para valores de $-1>k T>1$, siendo -1 correspondiente al caso RCP y 1 al caso LCP, se produce una inversión de signos en las constantes de propagación, lo que corresponde a medios denominados metamateriales. Este nuevo régimen de funcionamiento está actualmente siendo estudiado con más detalles y será objeto de una publicación futura.

El mismo fenómeno descrito anteriormente se repite cuando el medio tiene pérdidas $(\alpha \neq 0)$. En las Figs. 8 y 9 se puede observar que las variaciones de la potencia del quirosoliton en función del factor quiral mantienen su forma aunque la potencia ha disminuido por efecto de las pérdidas.

Como una consecuencia de lo anterior, se puede deducir que la variación del factor quiral permite compensaciones desde 0 hasta -1 para solitones RCP y desde 0 hasta 1 para solitones LCP. Por lo tanto, tomando como referencia un material aquiral $(k T=0)$, es posible aumentar la potencia de los pulsos que se propagan por el medio, y compensar las pérdidas de éste, eligiendo un factor quiral negativo para pulsos circularmente polarizados a la derecha (RCP) y factores quirales positivos para pulsos circularmente polarizados a la izquierda (LCP).

De las Figs. 8 y 9 se puede deducir, también, el efecto levemente anisotrópico que se tiene entre las figuras cuando se pasa de pulsos RCP a LCP modificando el signo del factor quiral. Por otra parte, para el caso de quirosolitones RCP es posible obtener incrementos de hasta $60 \%$ en la potencia cuando el factor quiral varía de 0 hasta $-0,7$ para un pulso sin atenuación e incrementos de hasta un $50 \%$ en el caso de tener una atenuación de $5 \mathrm{~dB}$. Se obtienen resultados similares para el caso de quirosolitones LCP, pero el factor quiral 
debe variar entre 0 y $+0,7$. Aquí se observa una leve anisotropía (comparar Figs. 6 y 7, y Figs. 8 y 9). Esto es típico del dicroismo mencionado anteriormente.

Estos resultados están normalizados de manera que son válidos tanto para microondas como para fibras ópticas para un mismo nivel de potencia de entrada requerido para la formación y propagación del soliton. El caso de quirosolitones ópticos de primer orden ya ha sido estudiado suficientemente para el caso sin atenuación [25], [26], en régimen isotrópico y anisotrópico, donde la justificación física acerca de su formación ha sido explicada satisfactoriamente. El caso de la formación de quirosolitones de microondas en medios bioplasmáticos merece una justificación más profunda en este trabajo. $\mathrm{Si}$ suponemos que el medio está compuesto de agua proteínica en la cual se encuentran agrupamientos de macromoléculas o cluster equipadas con dipolos eléctricos, que presentan una quiralidad geométrica, el campo eléctrico de la microonda interactúa con dichos dipolos haciéndolos rotar de su posición inicial, y el campo inducido en el interior del medio complejo se polariza elípticamente a la derecha o a la izquierda, La fuerza que actúa sobre los electrones produce una velocidad de arrastre de los iones que forman las macromoléculas. Al combinar la ecuación de continuidad con la ecuación de fuerza se tiene una ecuación ión-acústica, que es proporcional a la ecuación no lineal de Schrödinger (13), donde los perfiles de intensidad de los campos en las células pueden ser computados de (13), usando las condiciones de contorno apropiadas.

Si la difracción y el término no lineal son iguales, el haz se propaga sin divergir pero sufriendo un dicroismo, debido a la conductividad del medio bioplasmático. Para campos típicos de microondas, el valor crítico para la formación solitónica es del orden de $100 \mathrm{~V} / \mathrm{m}$, La densidad de electrones requerida debe ser de 10.000 electrones por célula. El término no lineal, que sigue aproximadamente una ley cuadrática, sería el responsable de la desmodulación de campos en el tejido celular. Este mecanismo justifica el hecho de suponer que dentro del tejido cerebral es posible la propagación de solitones quirales debido a la excitación de microondas de teléfonos celulares.

\section{CONCLUSIONES}

Se ha presentado el modelado y la simulación de pulsos ópticos o de microondas circularmente polarizados a la derecha (RCP) y circularmente polarizados a la izquierda (LCP) en un medio dispersivo, quiral y no lineal. Se demostró que las pérdidas que introduce el medio de propagación sobre pulsos solitónicos que viajan a su través, puede ser compensada variando el factor de quiralidad del medio de propagación, resultando quirosolitones RCP y LCP y obteniéndose, para rangos de atenuación de 0 y $5 \mathrm{~dB}$ y factores quirales de \pm 1 , compensaciones del orden del $50-60 \%$.

La bianisotropía observada en el cálculo numérico es interesante desde el punto de vista experimental como herramienta numérica para el estudio de propiedades ópticas y de microondas de tejidos con múltiple dispersión. Como una técnica no invasiva se puede predecir la polarización, ya que la retención del grado de polarización circular es más fuerte en medios que contienen moléculas ópticamente activas (por ejemplo la glucosa que es quiral tipo RCP). Esto podría ser importante en el monitoreo y detección de glucosa en la sangre.

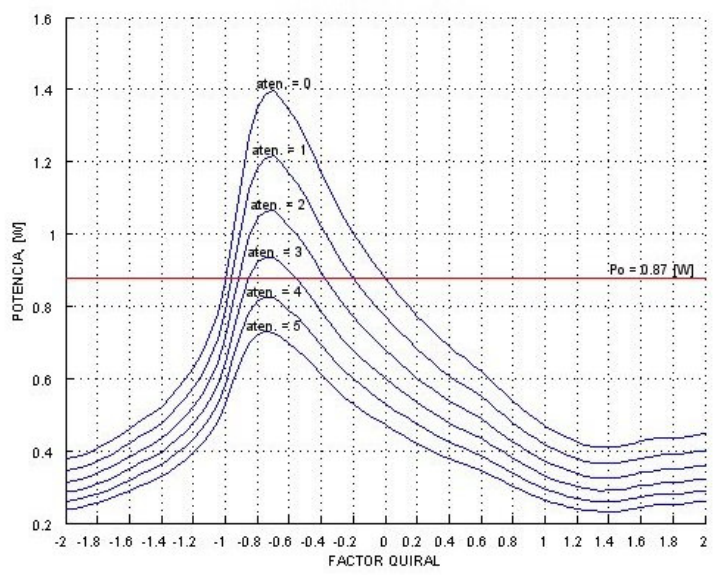

Fig. 8.- Potencia de quirosolitones RCP v/s factor quiral para atenuaciones de 0 a $5 \mathrm{db}$.

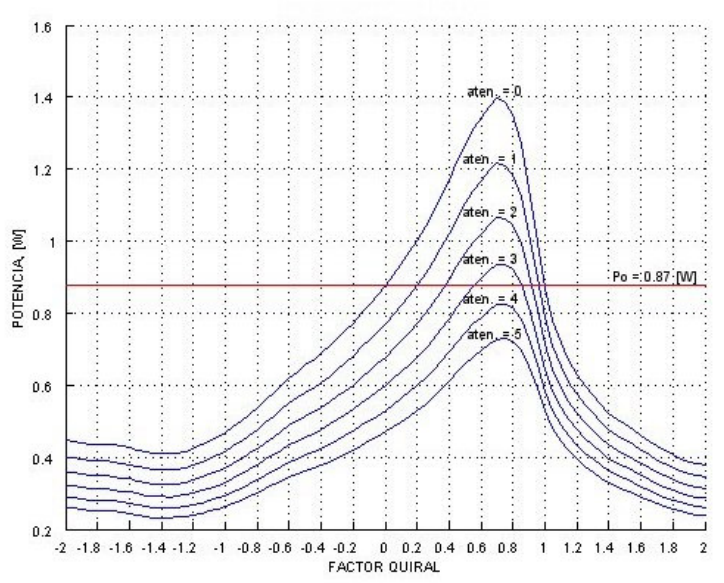

Fig. 9.- Potencia de quirosolitones LCP v/s factor quiral para atenuaciones de 0 a $5 \mathrm{db}$. 


\section{AGRADECIMIENTOS}

Este trabajo ha sido parcialmente financiado por los proyectos Fondecyt $\mathrm{N}^{\mathrm{o}} 1040744$ y UTA No 872103 y 8722-03.

\section{REFERENCIAS}

[1] Born and K. Hang, 'Dynamical Theory of Crystal Lattice", Clare ndon Oxford, 1954.

[2] V.M. Agranovich, V.L. Ginzburg, 'Crystal Optics with Spatial Dispersion and Excitons", Wiley, London, 1984.

[3] A. Lakhtakia, "Beltrami Fields in Chiral Media", Word Scientific, Singapore, 1994.

[4] I.V. Lindell, et al, 'Electromagnetic Waves in Chiral and Bi-isotropic Media", Artech House. Second Edition, 2000.

[5] A. Lakthakia; Selected Papers on Natural Optical Activity, SPIE Milestones Series Vol. MS515, SPIE, Bellingham, WA. 1991.

[6] H. Torres Silva, et al; 'Electromagnetic Properties of a Chiral-Plasma", Pramana Journal Physics 48, $1,1997$.

[7] H. Torres Silva; "Chiro-Plasma Surface Waves", Advances in Complex Electromagnetic Materials, Kruwer Academic Publishers, Netherlands, 249.

[8] M. Zamorano, H. Torres; 'Ecuación de Schrödinger para una Fibra Óptica Quiral”, Revista Mexicana de Física, 46 (1), pp. 62-66, Feb. 2000.

[9] M. Zamorano, H. Torres; 'Solution of Schrödinger equation for a nonlinear Kerr medium with linear chiral activity and cubic anisotropy using the splitstep Fourier method", Revista Facultad de Ingeniería, Vol. 8, Universidad de Tarapacá, Arica - Chile, 2001.

[10] A. Fresnel Ann Chim Phys 28.147 (1825); Oeuvers complets 1.731, Paris, 1866.

[11] P. Drude Lehrhuch der Optik (S Hirzel, Leipzig. 1900); English traslation: Handbook of Optics, Dover New York, 1959.

[12] L. Boltzmann Pogg Ann Phys Chem Jubelband, 128, 1984.

[13] K. F. Lindman Ann Phys Leipzig 63.621 (1920) 69.270, 1922.

[14] S.F. Mason; 'Molecular optical activity and the chiral discriminations", Cambridge University Press, Cambridge, 1982.

[15] L.D. Barron; "Molecular light scattering and optical activity", Cambridge University Press Cambridge, 1982.

[16] C. Oldano, M. Becchi; 'Natural potical activity and liquid crystals", Pramana-J. Phys., Vol 53, No 1 , pp. 131-143, July 1999.
[17] A. Lakhtakya, et al, "Time-Harmonic Electromagnetic Fields in Chiral Media", Lecture Notes in Physics 335, Springer-Verlag, 1985.

[18] G. Agrawal; 'Non Linear Fiber Optics", Academic Press, 1995.

[19] J.R. Smith, et al; 'Composite medium with simultaneously negative permeability and permittivity", Phy. Rev. Lett., Vol 48, No 18, pp. 4184-4187, May 2000.

[20] H. Born, et al; Bioelectromagnetics, Vol 18, pp. 187-189, 1997.

[21] H.F. Guttman; Bioelectrochemistry, Ed. F. Guttman and H. Keyzer, Plenum, New York, 1986.

[22] C. Henríquez; "Análisis y Simulación de la Propagación de Solitones Polarizados Circularmente en un Medio Quiral"; Memoria de Titulación Ing. Civil Electrónica, Universidad de Tarapacá, Arica-Chile, 2001.

[23] C.G. Parazzoli, et al, 'Experimental verification and simulation of negative index of refraction using Snell's law", Phys. Rev. Lett., Vol 90, 107401, 2003.

[24] R. Marquez, et al, 'Role of bianisotropy in negative permeability and left-handed metamaterials", Physical Review B, Vol. 65, pp. 144440/1-6, 2002.

[25] H. Torres, M. Zamorano; 'Chiral Effect on Optic al Soliton" The Journal Mathematics and Computers in Simulation, Vol. 62, pp. 149-161, 2003.

[26] M. Zamorano, H. Torres, 'Efecto de la quiralidad sobre solitones polarizados en un medio anisotropico", Rev. Mex. de Física, Vol. 49, (1) Feb. 2003. 